\title{
Evidence Based Treatment of Polycystic Ovarian Syndrome/Diseases (PCOS/PCOD) with Homoeopathy
}

\section{Rahul Singh*}

Senior Medical Advisor- Bhargava Phyto lab Pvt. Ltd, Care 'N Cure Homoeopathic Health Centre, Delhi, India

*Corresponding Author: Rahul Singh, Senior Medical Advisor- Bhargava Phyto lab Pvt. Ltd, Care 'N Cure Homoeopathic Health Centre, Delhi, India.

Received: September 16, 2019; Published: October 23, 2019

DOI: $10.31080 /$ ASMS.2019.03.0447

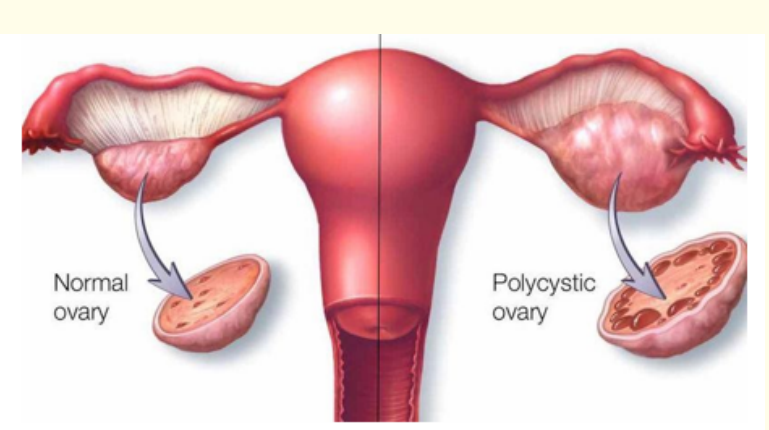

Figure 1

\section{Abstract \\ Polycystic Ovarian Syndrome/Diseases}

Polycystic ovarian syndrome (PCOS) or Polycystic ovarian diseases (PCOD), is a common endocrine system disorder among women of reproductive age. It is a condition in which a woman"s levels of the sex hormones estrogen and progesterone are out of balance. This leads to the enlarged ovaries that contain small collections of fluid (ovarian cysts), called follicles which can be located in each ovary. These cysts can be seen during an ultrasound exam. PCOS can cause problems with a women"s menstrual cycle, fertility, cardiac function, and appearance.

Keywords: Polycystic Ovarian Syndrome; Homoeopathy

\section{Background}

The syndrome was previously called Stein-Leventhal Syndrome after the physicians who first characterized it in the 1930s. Although its cause remains unknown. Hippocrates (460 - 377 B.C.) notes, But those women whose menstruation is less than three days or is meager, are robust, with a healthy complexion and a masculine appearance; yet they are not concerned about bearing children nor do they become pregnant. Soranus of Ephesus (c. 98 -138 A.D.) noted thatsometimes it is also natural not to menstruate at all. It is natural too in persons whose bodies are of a masculine type. we observe that the majority of those not menstruating are rather robust, like mannish and sterile women (Gynecology, Book I. Art. 23 and Book I. Art. 29). 


\section{Epidemiology and risk factors}

Prevalence estimates for PCOS, as defined by the NIH/NICHD criteria, indicate that PCOS is a common endocrinopathy affecting $4 \%-8 \%$ of women of reproductive age. Recently, several groups have demonstrated that the prevalence of PCOS varies depending on the diagnostic criteria used.

The family history of PCOS is a risk factor for PCOS. Based on the clustering of cases in families, PCOS is considered to be a heritable disorder. A high prevalence of PCOS or its features among first-degree relatives is suggestive of genetic influences.

\section{The cause of PCOS}

The exact cause of PCOS is unknown. doctors believe that hormonal imbalances and genetics play a role. Women are more likely to develop PCOS if their mother or sister also has the condition. Overproduction of the hormone androgen may be another contributing factor. Androgen is a male sex hormone that women"s bodies also produce. Women with PCOS often produce higherthan-normal levels of androgen. This can affect the development and release of eggs during ovulation. Excess insulin (a hormone that helps convert sugars and starches into energy) may cause high androgen levels [1-6].

\section{Symptoms of PCOS}

- Irregular menses (usually delayed)

- Weight gain and difficulty in losing weight

- Acne

- Dandruff

- $\quad$ Pain in Abdomen

- Excessive hair growth on the face, chest, back

- $\quad$ Thinning of hair

- Infertility

- Miscarriage

- High blood pressure

- Diabetes

- High cholesterol

\section{Diagnosis of PCOS}

There is no definitive test for PCOS. Diagnosis is depending on medical history and symptoms of patients. the physical and pelvic examination can help to find out the signs of PCOS.
Blood tests

- $\quad$ Thyroid function tests (TSH).

- $\quad$ Fasting glucose tests to measure blood sugar levels.

- $\quad$ Lipid level tests to assess the amount of cholesterol. USG (Ultra Sonography)

\section{Treatment of PCOS}

Treatment for PCOS is not curative as per modern science. It focuses on controlling and managing the condition to prevent complications. The treatment also varies from woman to woman, depending on their symptoms.

A healthy diet and regular exercise may help to control the PCOS, particularly in those women, who are overweight. This can help to regulate the menstrual cycle and lower the blood glucose levels.

Surgery can be the last option. A recent treatment option uses laparoscopy to treat patients with PCOD.

\section{Complications of PCOS}

- Hypertension (high blood pressure).

- High cholesterol.

- Anxiety and depression.

- Endometrial cancer (cancer caused by thickening of the lining of the uterus).

- Heart attack.

- Diabetes.

- Breast cancer.

Homeopathic management for PCOS

Polycystic Ovarian Syndrome (PCOS) can be treated by Homoeopathic medicines. Homoeopathic medicines are very safe and can cure PCOS permanently and without any side effect. The complete cure of PCOS is a time-consuming process and it cannot be expected in a few days. It requires complete observation regarding the change of symptoms in the patient and frequent clinical follow-ups. Here, we are presenting two cured cases of PCOS/PCOD treated by Homoeopathic medicines.

Case 1

A case of PCOD treated by homoeopathic medicine Pulsatilla

This is a case of a college going girl of 18 years old, came to me for the complaint of Amenorrhea for last 6 month and pain in right 
side of the abdomen. She came to me on 5th of February, 2016. Her mother is a regular patient of mine.

Associated problem.

Acne on face, Weight was increasing Past h/o: - Nothing specific Family H/o

- $\quad$ Father: - Hypertension, Renal calculi, Drink Alcohol regularly.

- Mother:PaininLegs_Personal H/o.

- Appetite: normal.

- Craving: Pickle.

- Thirst: Below average.

- Perspiration: only on exertion.

- Thermal: Hot, can't remain in a warm room, likes open air.

- Stool: Regular, Disturbed by oily and fastfood.

- Urine: normal.

- Sleep: normal.

- Fear:Ofghost,Dark,Height_Mental.

- Get irritable on small thing.

- Weeping mood.

- According to her mother, she is very sweet by nature and obeys her instructions most of the time Menstrual H/o.

- Menarche: at age of 14 years.

- Much pain in abdomen before and on the first day of menses.

- Amenorrheaforlast6 month Investigations.

We advised her to get an Ultrasonography of Lower Abdomen. According to her USG report, she was having Poly Cystic Ovarian disease in her Right ovary.

Treatment

After proper analysis of her symptoms, she was given PULSATILLA 0/1 BD. After two months she had her menses with less pain. Now mentally she is calm and her acne was also disappearing. Follow -up: PULSATILLA 0/1 refill BD. Follow -up: PULSATILLA 0/2 BD. Follow -up: PULSATILLA 0/2 refill BD. Intercurrent remedy: Thujha $1 \mathrm{M}$ - one dose. After about six months, she was normal with regular menses. Her USG report confirms it, as a report was normal.

Both USG reports are attached.
Before Treatment

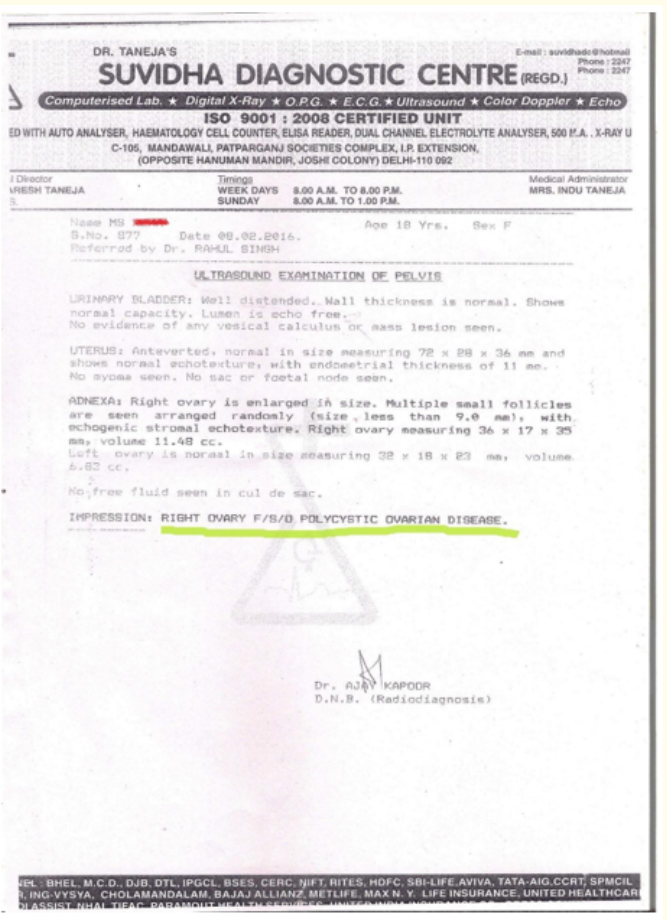

Figure 2

After Treatment

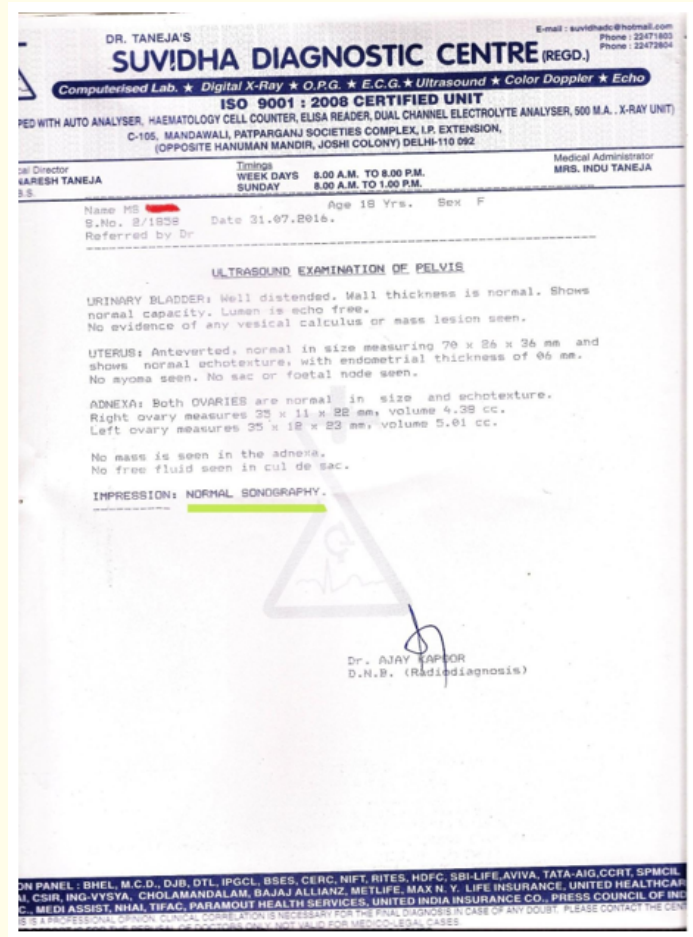

Figure 3 


\section{Case 2}

This is a case of a 30 years old lady, Mrs. N.K, having problem of "irregular and painful menses" for last 1 year. After doing USG whole abdomen, she was told of having PCOS, by her gynecologist. She refused to start hormone therapy and came to us for homoeopathic treatment as previously she was cured by our homoeopathic medicines for her facial "flat warts". After proper assessment of her case, we started homoeopathic medicine to her. Within three and half months, her USG report was normal for PCOS.

\section{Associated problem}

Hair fall, obesity and indigestion

Past h/o: - Facial warts (Flat warts - cured by homoeopathy) Family H/o

- Father: - Hypertension.

- $\quad$ Mother: Pain in knees Personal H/o.

- $\quad$ Appetite: Normal.

- Craving: Spicy food and non-veg

- Thirst: Normal.

- $\quad$ Perspiration: Profuse, mainly on head.

- Thermal: Normal.

- Stool: Hard, mostly constipated.

- Urine: Normal.

- $\quad$ Sleep: Normal.

- Fear: Of going alone Mental.

- Lack of confidence.

- Yielding in nature, friendly.

- $\quad$ Slow acting Menstrual H/o.

- Menarche: at age of 13 years.

- Irregular for last 1 year.

\section{Investigations}

As per her USG report, she was having PCOS with small nabothian cyst in cervix.

\section{Treatment}

As per her symptoms, she was given Calcarea carbonica 30 tds and Apis mellifica 200 weekly one dose.

By next month, she was having regular menses. After about 3 months, she was advised for another USG. Her USG report was normal. Both USG reports are attached.
Before Treatment

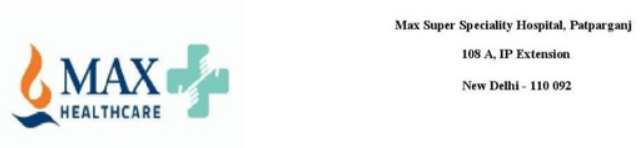

INVESTIGATION REPORT

\begin{tabular}{|llll|}
\hline Patent Name & Location & $:$ Patparganj \\
Agelsex & $: 30$ Year(s)Female & IPNo & $: 0$ \\
Maxid & $: 4$ & Order Date & $: 17 / 07 / 2018$ \\
Ret. Doctor & $:$ cyls & Report Date & $: 17 / 072018$ \\
\hline
\end{tabular}

USG Whole Abdome

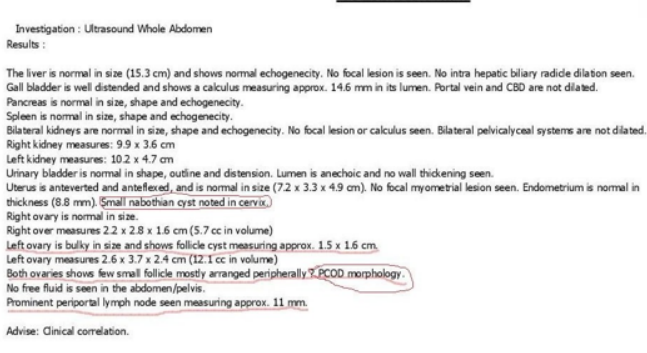

Figure 4

\section{Before Treatment}

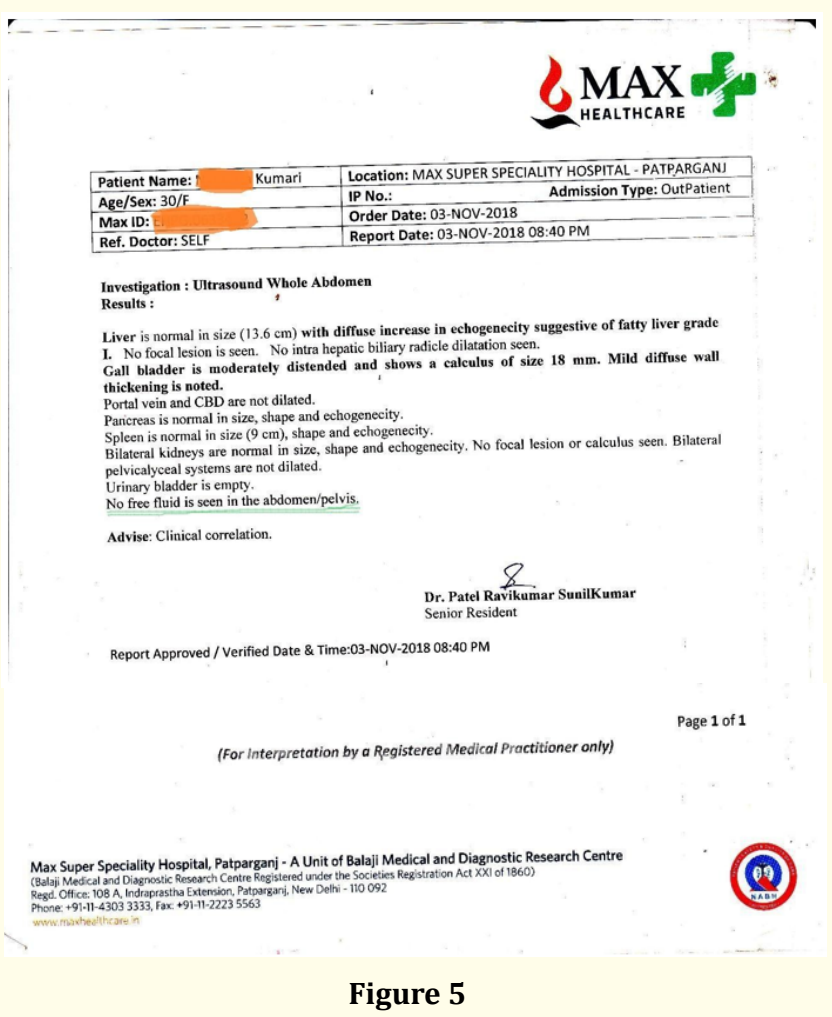




\section{Conclusion}

PCOS is the most common but complex disorder, which affects many women of reproductive age. As a syndrome, it has multiple health issues and reason of most common form of anovulatory infertility, with long-term health concerns that cross the life span. PCOS is also associated with insulin resistance and a high prevalence of obesity. Treatment for PCOS is not curative as per modern science. It focuses on controlling and managing the condition to prevent complications. The treatment also varies from woman to woman, depending on their symptoms. Homoeopathy is very effective for treating polycystic ovarian syndrome (PCOS). With homoeopathy, we can also avoid the complications of PCOS and restore the health of the reproductive women.

\section{Bibliography}

1. Shaw's Textbook of Gynecology 16th edition

2. Dc Dutta'S Textbook of Gynecology 7th edition

3. Repertory of the Homoeopathic Materia Medica by Dr. J. T. Kent, B. Jain Publishers, Reprint edition, (1998).

4. New Manual of Homoeopathic Materia Medica and Repertory by Dr. William Boericke, MD, B. Jain publishers

5. Skinner T. "The Diseases of Females”. New Delhi: B. Jain (P) Ltd.; (1997): 131

6. Allen HC and Genitalia Female HC. "Allen's Key Notes and Characteristics with Comparisons with Bowel Nosodes". New Delhi: B. Jain (P) Ltd (2004): 295-296.

\section{Volume 3 Issue 11 November 2019}

\section{(C) All rights are reserved by Rahul Singh.}

